\title{
Study on Insulation of Middle Joint of Power Supply Line of High Speed Railway
}

\author{
Fei Jia ${ }^{a}$, Xiaodong Zhang ${ }^{b}$, and Dandan Liu ${ }^{c}$ \\ School of Electrical Engineering, Beijing Jiaotong University, Beijing 100044, China. \\ a15121418@bjtu.edu.cn, bxdzhang@bjtu.edu.cn, c15121435@bjtu.edu.cn
}

Keywords: cable joint, finite element method, electromagnetic-thermal-mechanical coupling fields, aging of insulation material.

\begin{abstract}
Since the thermal expansion coefficient of the insulating material and the metallic material in the cable joint different is large, when the temperature changes, there is great stress in the insulating material or the tightening of the insulating material and the metal material, causing damage to the insulation, and the temperature too high will damage the thermal stability of the material, resulting in insulation damage, seriously affecting the safe operation of high-speed trains. In order to improve the reliability of power supply, finite element method is used to analyze the distribution characteristics of temperature field and stress field of cable joint in the process of normal and insulated aging. The study shows that the insulation state of cable joint can be judged by detecting the surface temperature and strain of cable joint, and it can provide a reference for the maintenance and maintenance of the cable head.
\end{abstract}

\section{Introduction}

The high speed railway contact network adopts $27.5 \mathrm{kV}$ single phase AC single core XLPE high voltage cable transmission, its safe operation has important significance [1]. There are many factors for the cable joint failure, but the analysis and summary find that the cable joint fault is mostly caused by the damage of the insulation material. The insulation status of power cable joint is closely related to the temperature, when the cable is in normal operation state, the maximum working temperature of the cable conductor is $90^{\circ} \mathrm{C}$, which has little effect on the insulation aging speed of the power cable connector; But when the cable will be in abnormal operation condition, the joint temperature exceeds the maximum temperature of the cable conductor during short circuit, will cause the cable insulation accelerated aging, even will happen thermal breakdown $[2,3]$. The study found that when the operating temperature of the XLPE cable exceeds $8 \%$ of the maximum operating temperature, its life will be reduced by halves; when the working temperature exceeds $15 \%$ of the maximum allowable working temperature, the cable life will only be $1 / 4$ of the original $[4,5]$.

The cable joint is the weakest link in the safe operation of the cable. The cable connector is made of various materials, and their respective expansion coefficients are different. The insulation material are often bonded with metal materials, because the difference of the expansion coefficient is very large, when the temperature changes, the internal insulating material or insulating material and metal material combination will produce high stress, causing the insulation damage; moreover, the high temperature will destroy the thermal stability of the material, cause insulation breakdown, seriously affect the safe operation of high-speed train.

Based on the study of cable joint structure, using finite element analysis software ANSYS to establish cable joint of electromagnetic-thermal-mechanical coupling model. The distribution characteristics of temperature field and stress field of cable joint in normal operation and aging of insulation layer are simulated and analyzed.

\section{Structural Model of Cable Joint}

The TYJV $1 * 150 \mathrm{~mm} 2$ high-voltage cable and cold shrinkable straight connector selected for simulation analysis. According to the data, the material used in each part of the cable joint is as 
follows: the material of the conductor core is copper, the conductor shielding material is peroxide cross-linked material, the connecting pipe material is copper, the insulating material is conductive silicone rubber, shielding material for copper, outer sheath material for polyethylene. The crosssection of the cable joint structure is shown in Fig. 1 the material properties of each part of the joint are shown in table 1.

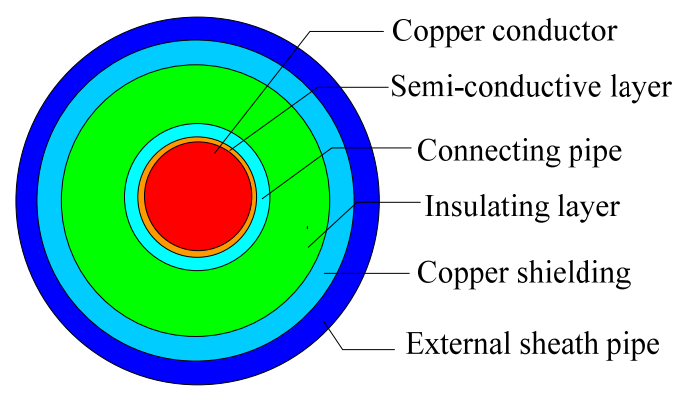

Fig. 1 Structure of cable joint

Tab. 1 Material properties of each part of the model

\begin{tabular}{ccccccc}
\hline Denomination & $\begin{array}{c}\text { Relative } \\
\text { permittivity }\end{array}$ & $\begin{array}{c}\text { Resistivity/ } \\
\Omega \cdot \mathrm{m}\end{array}$ & $\begin{array}{c}\text { Thermal } \\
\text { conductivity / } \\
\mathrm{W} \cdot \mathrm{m}^{-1} \cdot \mathrm{k}^{-1}\end{array}$ & $\begin{array}{c}\text { Elastic } \\
\text { modulus/ } \\
\mathrm{MPa}\end{array}$ & $\begin{array}{c}\text { Poisson's } \\
\text { ratio }\end{array}$ & $\begin{array}{c}\text { Coefficient of } \\
\text { thermal } \\
\text { expansion } / 10^{-} \\
5{ }^{\circ} \mathrm{C}^{-1}\end{array}$ \\
\hline $\begin{array}{c}\text { Copper } \\
\text { conductor } \\
\text { Semi- }\end{array}$ & 1 & $1.75 \mathrm{e}^{-8}$ & 400 & 121000 & 0.33 & 1.8 \\
$\begin{array}{c}\text { conductive layer } \\
\text { Connecting pipe }\end{array}$ & 100 & 0.168 & 0.48 & 34.23 & 0.48 & 28 \\
$\begin{array}{c}\text { Insulating layer } \\
\text { Copper } \\
\text { shielding }\end{array}$ & 3.1 & $1.75 \mathrm{e}^{-8}$ & 400 & 121000 & 0.33 & 1.8 \\
$\begin{array}{c}\text { External sheath } \\
\text { pipe }\end{array}$ & 1 & $1.75 \mathrm{e}^{-8}$ & 400 & 121000 & 0.33 & 1.8 \\
\hline
\end{tabular}

\section{Mathematical Model of Multiphysics Coupling Field for Cable Joint}

The essence of Multiphysics field coupling is the information transfer between multiple physical fields. The running process of the cable joint includes electromagnetic field, temperature field and stress field, and the interaction of these three physical fields is a Multiphysics field coupling problem. The coupling relation between the three physical fields of the cable joint includes the following two cases:

(1) The coupling between the electromagnetic field and the temperature field is described as follows: when distribution of the temperature field is solved by the heat conduction control equation, the heat source term in the equation is known first, and the heat source is determined by the electromagnetic loss density calculated in the electromagnetic field.

(2) The coupling between the temperature field and the stress field is described as follows: calculating the stress distribution of the cable joint model, the temperature distribution of the cable joint model needs to be obtained first, and the stress changes lead to deformation of cable joint structure, will also affect the calculation results of cable joint temperature field.

\subsection{Mathematical Model of Electromagnetic Field of Cable Joint}

The exact solution of electromagnetic problems, usually only according to the specific circumstances of the given initial and boundary conditions for solving the electromagnetic field problem, its solution with numerical solutions, the finite element method is one of the most effective and the most widely used numerical calculation method [6]. In order to simplify the calculation of 
electromagnetic field, the introduction of some basic assumptions: cable joint operation in the power frequency $(50 \mathrm{~Hz})$, ignoring the displacement current; cable joint parts materials are regarded as an isotropic homogeneous medium, and the physical parameters are constant; no free charge in the field.

Based on the above assumptions, according to the Maxwell equations, the vector magnetic potential is introduced. For the current region with an additional excitation source, the governing equation of the magnetic vector position is [7]:

$$
\left(\nabla \cdot \frac{1}{\mu} \nabla\right) \vec{A}=-\vec{J}_{s}+\varepsilon \frac{\partial^{2} \vec{A}}{\partial t^{2}}
$$

For the current region without an external excitation source, the governing equation of the magnetic vector position is:

$$
\left(\nabla \bullet \frac{1}{\mu} \nabla\right) \vec{A}=0
$$

Where, $\mu$ is the magnetic permeability, A is the vector magnetic field, $\varepsilon$ is the dielectric constant, and $\mathbf{J}_{\mathrm{s}}$ is the applied current density; when there is no applied current, $\mathrm{J}_{\mathrm{s}}=0$.

The vector magnetic potential is rapidly attenuated in the outer space of the cable conductor, so the vector magnetic potential at a certain distance from the cable surface is about zero, i.e., the vector magnetic potential of the outer boundary condition of the air field is zero.

\subsection{Mathematical Model of Temperature Field of Cable Joint}

For the temperature field analysis problem of the cable joint, the control equation of the steadystate temperature field can be obtained according to the law of conservation of energy and the law of Fourier heat conduction, which can be written in Cartesian coordinate system [8]:

$$
\frac{\partial^{2} \tau}{\partial x^{2}}+\frac{\partial^{2} \tau}{\partial y^{2}}+\frac{\partial^{2} \tau}{\partial z^{2}}+\frac{\partial \dot{\Phi}}{\lambda}=0
$$

Where, $\Phi$ is the thermal energy of the heat source; $\lambda$ is the thermal conductivity; $t$ is the temperature of the object.

The boundary condition of the temperature field of the cable joint is the temperature of the surrounding environment and the convective heat transfer coefficient between the outer sheath and the surrounding air. Usually the air temperature is set to $20^{\circ} \mathrm{C}$, air convection heat transfer coefficient of $12.5 \mathrm{~W} /\left(\mathrm{m}^{2} \cdot{ }^{\circ} \mathrm{C}\right)$.

\subsection{Mathematical Model of stress Field of Cable Joint}

Because the metal shielding layer is very thin, in order to simplify the calculation, the stress changes caused by electromagnetic force can be neglected. The analysis of the thermal stress field of the power cable joint structure can be described by the following equations:

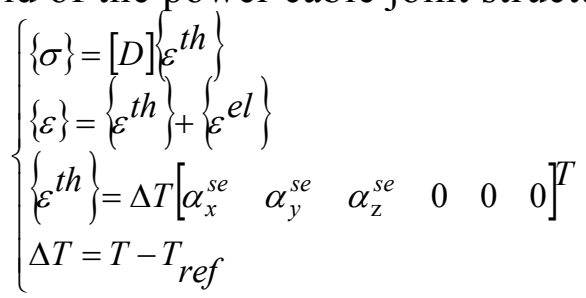

Where, $\sigma$ is the stress tensor; D is the strain coefficient; $\varepsilon$ is the total strain tensor; $\varepsilon^{\text {th }}$ is the thermal strain component; $\varepsilon^{\text {el }}$ is the elastic strain component; $\alpha^{\text {se }}$ is the coefficient of thermal expansion; $\Delta \mathrm{T}$ is the amount of temperature change compared to the reference temperature; $\mathrm{T}$ is the object temperature; $\mathrm{T}^{\mathrm{ref}}$ is the reference temperature.

Considering the radial heat transfer at the cable joint, the displacement on the cross section of the cable joint is considered to be the radial direction, that is, the axial displacement component is equal to zero. The boundary condition of the cable joint surface is the free boundary, i.e. there is no constraint on any external force and displacement. Simulation Analysis of Cable Joint

According to the research needs, distribution of the temperature field and stress field of cable joints under different conditions are simulated and analyzed by using ANSYS software. The simulation 
process is as follow: modeling the cable joint structure, meshing the finite element, load loads and boundary conditions, and finally solving the problem.

\subsection{Temperature and Stress Distribution of Cable Joints Under Normal Conditions}

Fig. 2 shows the temperature and stress distribution of the cable joint under normal running condition.

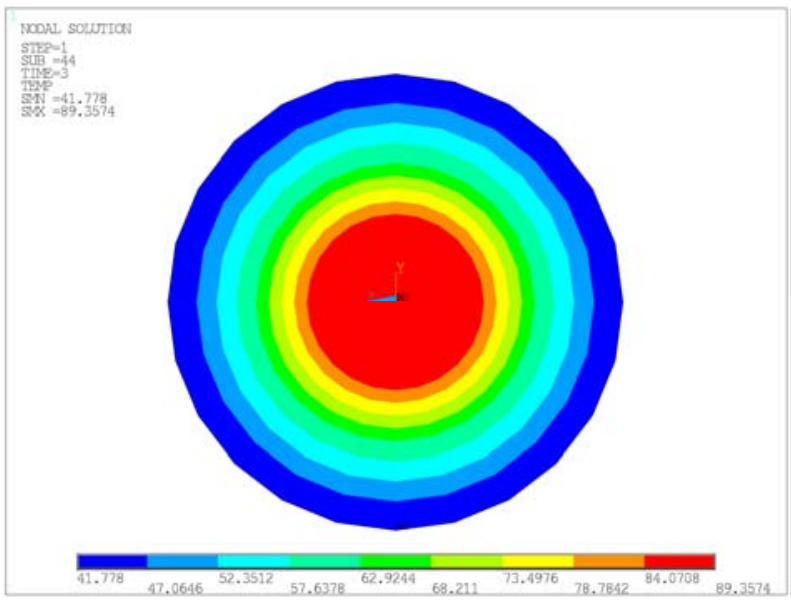

(a) Temperature distribution of cable joint

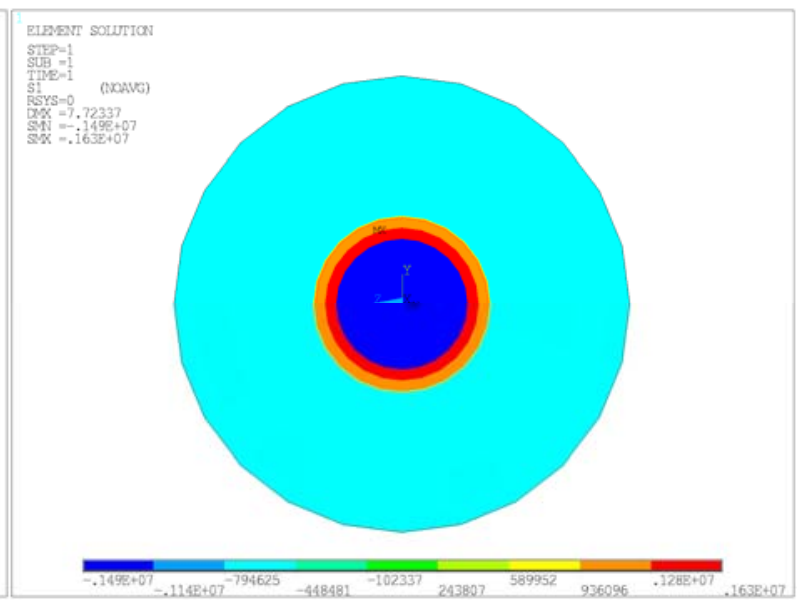

(b) Stress distribution of cable joint

Fig. 2 Distributions of temperature \& stress field of cable joint during normal operation

As shown in figure 2 (a), the maximum temperature at the normal work of the cable intermediate connector is $89.3574^{\circ} \mathrm{C}$. When the cable is in normal operation, the maximum working temperature of the cable conductor is $90^{\circ} \mathrm{C}$, and the result is normal. From figure 2 (b) shows that the connecting pipe and the semi-conductive layer at the junction of the maximum stress, followed by the insulating layer and the connecting pipe junction, the interior of the insulating layer, which is due to the cable insulation material and metal material thermal expansion coefficient difference is too large results.

\subsection{Temperature and Stress Distribution of Cable Joints during Aging of Insulating Materials}

Because the aging of the insulating material will decrease with the resistivity of the material, the aging of the material is simulated by changing the resistivity of the insulating material at the interface of the insulating layer and the stress cone during the simulation. At the interface, six different insulation resistors are set, as shown in table 2.

Tab. 2 the resistivity of the insulation material on the interface

\begin{tabular}{ccccccc}
\hline Interface & 1 & 2 & 3 & 4 & 5 & 6 \\
\hline Resistivity $/ \Omega \cdot \mathrm{m}$ & $3 \mathrm{e}^{8}$ & $2 \mathrm{e}^{8}$ & $1.5 \mathrm{e}^{8}$ & $5 \mathrm{e}^{7}$ & $2 \mathrm{e}^{7}$ & $1.8 \mathrm{e}^{7}$ \\
\hline
\end{tabular}

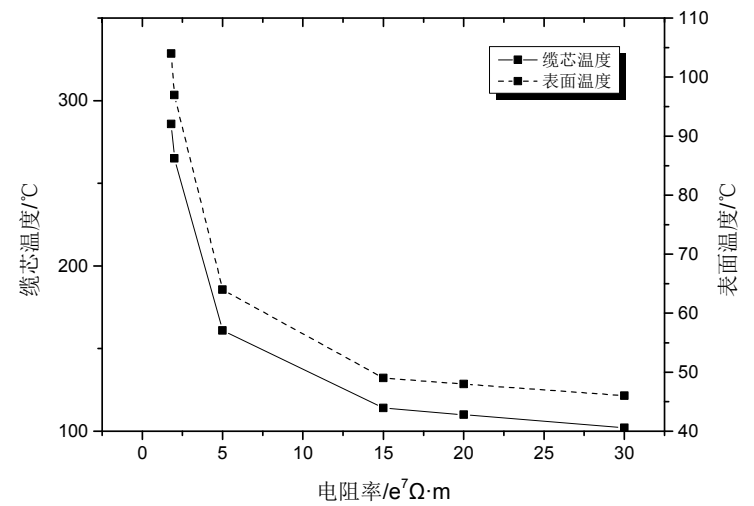

Fig. 4 Simulation temperature of cable joint

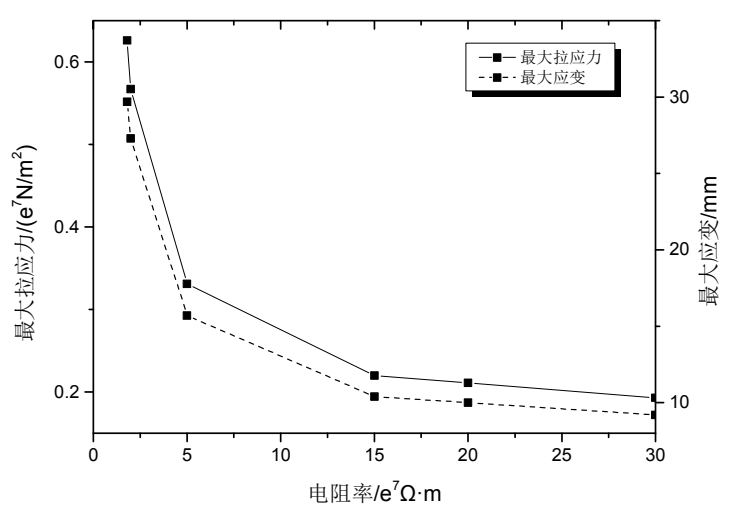

Fig. 5 Maximum tensile stress and strain of cable joint

Figure 4 shows the simulation temperature of the cable connector at different resistances at the interface. Figure 5 shows the maximum stress and strain of the cable joint at different resistances at the interface

The curve in figure 4, 5 shows that: 
(1) The surface temperature of the cable joint increases with the increase of the temperature of the cable core, and the change trend is consistent. It shows that the change of the cable core temperature can well reflect the change of the cable surface temperature.

(2) The maximum strain of the cable joint increases with the tensile stress it receives, and the change trend is consistent. It shows that the strain change of the cable joint can well reflect the stress change of the cable joint.

(3) With the development of insulation aging at the interface, there is a significant "transition period" between the surface temperature and the strain curve of the cable joint. Before the transition period, the temperature and strain change smoothly, and after the transition period, the temperature and strain increases rapidly.

\subsection{Judgment of Insulation Damage}

It can be seen from the above analysis that by detecting the surface temperature and strain of the cable joint is an effective way to judge the insulation damage of the cable joint. With the development of the insulation aging at the interface, there is a significant "transition period" between the surface temperature and the strain curve of the cable joint, this "transition period" can be seen as the cable connector insulation damage "early warning period", once the power cable into the "early warning period" means that the cable insulation is about to enter the rapid aging period, should take measures to suppress the cable connector Insulation is further aging.

In order to better predict the insulation damage, on the basis of the curve between temperature and strain of the cable joint and the above analysis, the surface temperature and strain of the cable joint are selected as the characteristic signal to judge the insulation damage of the cable joint, and the insulation of the cable joint is damaged make the following two criteria:

(1) Warning discriminant value. Select the surface temperature of $49{ }^{\circ} \mathrm{C}$, the strain is $10.4 \mathrm{~mm}$ as a cable connector into the "transition period" of the warning value. It is concluded that the cable joint insulation has been developed into a "transition period" through the "stable development period "of the aging at this moment. The staff should carry out maintenance and repair of the cable head in time.

(2) Determination value of insulation damage. From the above simulation and analysis, we can see that with the aging of the insulation, the rapid increase of the surface temperature and strain of the cable joint indicates that the cable insulation enters the "dangerous period". Therefore, the criterion of insulation damage should be determined in the "dangerous period" early, that is, the surface temperature and strain began to increase rapidly, therefore, select the surface temperature of $97{ }^{\circ} \mathrm{C}$, strain is $27.3 \mathrm{~mm}$ as a cable insulation damage criteria. When the cable insulation enters the "hazardous period", the worker should change the connector in time.

\section{Summary}

The finite element analysis software of ANSYS was used to simulate the distribution of the temperature field and the stress field of the cable joint during the normal and continuous aging of the cable joint. The research shows that the staff can judge the aging state of the joint insulation according to the change of the surface temperature and strain of the cable joint, and maintain and maintain the cable joint in time.

\section{References}

[1]. Jun Deng, Qingdong He. High speed electrified railway $27.5 \mathrm{kV}$ traction power supply cable insulation state pre-maintenance live detection technology application [J]. world rail transit, 2013, (7): 241-246.

[2]. GB/T 28427-2012, 27.5kV single phase AC XLPE insulated cables and accessories for electrified railway $[\mathrm{S}]$.

[3]. K e Tang, Wen Wu, Junjie Ding et al. Simulation of cable joint temperature field based on finite element method [J]. Electric power construction, 2016,37 (2): 145-150. 
[4]. Yanming Yang. Calculation of ampacity of power cable based on finite element method [D]. Harbin: Harbin University of Science and Technology, 2012.

[5]. Pan Luo, Zhigang Ren, Yang Xu et al. analysis of aging high pressure XLPE cable insulation aging state [J]. Journal of electrical technology, 2013, 28 (10): 41- 46.

[6]. Longhan Xie, Yu Geng, Wan Qiu. ANSYS electromagnetic field analysis [M]. Beijing: Electronic Industry Press, 2012.

[7]. Qizheng Ye, Dezhi Chen. Electromagnetic field course [M]. Beijing: Higher Education Press, 2012.

[8]. Zepeng Wang, Xiuhui Zhang, Renxi Hu. ANSYS 12. 0 thermodynamics finite element analysis from getting started to proficient [M]. Beijing: Mechanical industry press, 2010.6. 